abelian codes in this context. Chapter 5 gives a survey of the theory of group representations from the linear algebra point of view, and the last chapter utilizes this theory to construct codes for the Gaussian channel.

Only an introductory knowledge of modern algebra is needed to understand this book. The topics mentioned above are all developed and most of the theorems are proved. In addition, at the end of each chapter there are interesting exercises, many of which advance the theory. The more difficult exercises contain references. Comments are provided at the end of each chapter and an extensive set of references are at the end of the book. This book should provide a valuable tool for those mathematicians who have recently become interested in investigating some of the open problems in error-correcting codes as well as those already in the field. Areas requiring further investigation are noted in this book. This book could be a basis for an advanced undergraduate or graduate course in combinatorics, coding theory, or applications ${ }^{\circ}$ of modern algebra.

\title{
Vera Pless
}

Braids, links, and mapping class groups, by Joan S. Birman, based on lecture notes by James Cannon, Annals of Mathematics Studies, No. 82, Princeton University Press, Princeton, New Jersey, 1975, $228+$ ix pp., $\$ 8.50$.

Talleyrand is supposed to have said that nobody could know the full sweetness of life who had not lived before the French Revolution. One may say that nobody can know the full charm of topology who had to learn it after it became rigorous. Artin's first paper (published in 1925) on the theory of braids is a perfect and lasting monument of this charm. It is a paper containing almost exclusively ideas and results but practically no machinery. Birman's monograph gives a nearly complete account not only of Artin's results but also of the numerous important applications, later developments and generalizations of the theory of braids, many of which are due to the author. Her presentation is, of course, completely rigorous, but it is remarkable that she has been able to preserve much of the appeal to geometric intuition which helped to make Artin's paper so attractive.

The book is written in a concise but lucid style. The prerequisites are a solid knowledge of basic algebraic topology and familiarity with the elements of the theory of presentations of groups. Many more specialized concepts and theorems (as, for instance, the free differential calculus of $\mathrm{R}$. Fox), are developed in detail and sometimes with new proofs. For some theorems, only an outline of the proof or only a survey is given. These cases are fully covered by references to the literature.

The first chapter begins with the definition of the pure (or unpermuted) braid group of a manifold $M$ of dimension $>2$ as the fundamental group of the space

$$
F_{0, n} M=\left\{\left(z_{1}, \cdots, z_{n}\right) \in \prod_{i=1}^{n} M / z_{i} \neq z_{j} \quad \text { if } i \neq j\right\}
$$


where $\left(z_{1}, \cdots, z_{n}\right)$ stands for an ordered $n$-tuple of points and the product is the $n$-fold product space of $M$. By indentifying $n$-tuples which differ only by a permutation of the points $z_{1}, \cdots, z_{n}$, we obtain a space $B_{0, n} M$ whose fundamental group is the full $n$th braid group of $M$. In the case where $M$ is the euclidean plane, the group thus defined is Artin's braid group. Artin's geometric definition is recovered from the one given above by a simple argument. (However, the latter definition appears first, in the special case where $M$ is the two-sphere, in a paper by Hurwitz which was published in 1891. There the resulting groups are called "monodromy groups".) Subsequently, the author derives the results of Artin's papers of 1925 and 1947 with elegant and rather recent arguments, describing both the algebraic and the geometric aspects of the braid groups $B_{n}$ of the plane and also of the two-sphere in detail, and indicating the generalizations introduced by $D$. Goldsmith. The braid groups of other two-manifolds are covered merely by references. That higher-dimensional manifolds cannot be expected to produce very interesting braid groups is indicated by a theorem (due to the author) according to which the braid groups of $M$ are merely Cartesian powers of its fundamental group if $M$ is closed, smooth, and of dimension $>2$. Altogether, the first chapter may be described as a brief, lucid and modern introduction to the fundamental properties of the braid groups $B_{n}$ of the plane which contains the results of Artin's papers, giving a simplified and elegant outline of the proofs and a survey of the generalizations which have developed since the publication of Artin's work.

The second chapter contains the first complete and rigorously proved answer to a problem raised by Artin which has established the importance of the theory of braids for the theory of links.

Using a theorem due to Alexander, Artin had observed that the group of any tame link (i.e. the fundamental group of the space arising from euclidean 3-space by removal of a tame link) can be presented in terms of finitely many generators and relators by describing the link as a closed braid. Artin's presentation of the groups of links has both algebraic and geometric advantages. It also raised the possibility of a complete classification of links, describing every one uniquely by a finite set of data. Obviously, conjugate elements of $B_{n}$ define braids which, if closed, produce the same link. Therefore, solving the conjugacy problem in $B_{n}$ is a prerequisite of carrying out Artin's program. A solution of the conjugacy problem was obtained by Garside in 1969, and his solution (with simplifications by the author) is given in Chapter 2. However, it has been known for some time that the conjugacy classes of $B_{n}$ are not in one-one correspondence with topologically different links and also that the same link may be represented as a closed braid with different values for the number $n$ of strands of the braid. In 1945, A. A. Markov sketched a proof of the fact that any two presentations of a link as a closed braid can be carried into each other by finitely many elementary changes of finitely many types, such that each of these changes preserves the form of the link as a closed braid (although not necessarily the number of strings). The first full and detailed proof of Markov's Theorem is given in 
Chapter 2, which now provides a foundation for the implementation of Artin's program (which, however, still faces formidable difficulties). The last section of Chapter 2 describes most of the progress that has been made so far. It ends with the formulation of a theorem found by Burau in 1936, the proof of which requires the theory of matrix representation of $B_{n}$.

This theory is the topic of Chapter 3. It starts with an outline of the free differential calculus developed by R. Fix which, per se, is a useful introduction into this theory.

Since Artin had found a faithful representation of $B_{n}$ as a group of automorphisms of a free group, it is rather easy to find finite-dimensional matrix representations for $B_{n}$. However, the literature so far has been scattered and not coordinated, and the streamlined account of the theory by Birman (with several simplifications and new results found by her) will be highly welcome to anybody working in the field. To give details appears to be impossible without introducing a body of formulas which would be out of place in a general review.

Chapter 4 deals with the mapping class groups of two-dimensional orientable manifolds of arbitrary genus $\mathrm{g}$ which are either closed or derived from a closed manifold by the removal of a finite number of points. To define these groups algebraically (i.e. by a presentation in terms of generators and defining relations) is an old problem which goes back to the work of Hurwitz on Riemann surfaces and of Fricke (1891) on Fuchsian groups. Interest in this problem has been renewed by the recent advances in the theory of moduli of Riemann surfaces and of Kleinian groups. Its connection with braid groups was first observed by the reviewer in 1934 in the case of genus zero. However, the relationship for $\mathrm{g}>0$ is much more complex. It uses the realization of a surface of genus $g$ as branched coverings of the sphere. The theory has been developed mainly by the author, partly in cooperation with $\mathrm{H}$. Hilden. A particularly striking result is the presentation of the mapping class group of a closed orientable two-dimensional manifold of genus 2 as a quotient group of $B_{6}$, with a proof which provides a full understanding of the reasons for the connection between the two groups. Again, an account of the many results of this chapter would require the development of an extensive body of notations and will therefore be omitted. However, it should be noted that a full account of the basic topological facts is given in this chapter. There are no loose ends, i.e. no references to "well-known" facts, and, in particular, the theory of Dehn's "twists" is developed carefully and rigorously.

Chapter 4 ends with a survey of the theory of mapping class groups of 2-manifolds and with comments on the importance of this theory for the construction of 3-manifolds.

The last chapter, entitled Plats and links discusses a rather recent and promising algebraic-topological connection between Artin's braid groups and links in 3-space. A plat arises from a braid with $2 \mathrm{~m}$ strands by connecting the $(2 k-1)$ st and the $2 k$ th upper endpoints and also the corresponding lower endpoints of the braid for $k=1,2, \cdots, m$. The fundamental 
theorem states that all links may be represented as plats. Again, details of the emerging theory cannot be described here.

An appendix lists 34 research problems of various and, as admitted by the author, partly considerable degrees of difficulty. These give a very good idea of both the complexity and the promise of the existing theory.

The list of references is excellent. It is not arranged in the usual manner by authors but by the years of publication, a style introduced by Ralph Fox.

In summary it may be said that this monograph is an excellent guide to a field of research which is particularly attractive because it involves several mathematical disciplines. It has been planned with great care, and the introductions to the various chapters clearly outline the motivating ideas. It is perhaps not a textbook, but it is a very good basis for a seminar.

W. Magnus

Infinite-dimensional Lie algebras, by Ralph K. Amayo and Ian Stewart, Noordhoff International Publ., Leyden, The Netherlands, 1974, 425+xi pp.

Is there a theory of infinite-dimensional Lie algebras? Finite-dimensional Lie algebras over $\mathbf{R}$ or $\mathbf{C}$ (and even over p-adic fields) are forced upon us almost as soon as we begin to think about Lie groups; the introduction of linear algebraic groups and related finite groups then makes it natural to allow even more general base fields. Infinite-dimensional Lie algebras, on the other hand, have arisen only sporadically and have been investigated only in special settings, e.g.: free Lie algebras (cf. N. Bourbaki, Groupes et algèbres de Lie, Chapter 2, Hermann, Paris, 1972); simple algebras of infinite type (E. Cartan, S. Sternberg, V. Guillemin, V. G. Kac); Lie algebras of formal vector fields (I. M. Gel'fand, D. B. Fuks); Banach-Lie algebras (cf. P. de la Harpe, Lecture Notes in Math, vol. 285, Springer, Berlin, 1972); Lie algebras defined by generalized Cartan matrices (V. G. Kac, R. V. Moody).

Amayo and Stewart concentrate entirely on certain algebraic aspects of infinite dimensional Lie algebras, emphasizing in their preface the "surprising depth of analogy" between these and infinite groups, but adding: "This is not to say that the theory consists of groups dressed in Lie-algebraic clothing. One of the tantalising aspects of the analogy, and one which renders it difficult to formalise, is that it extends to theorems better than to proofs." This seems a fair assessment.

Their book has 18 chapters, which to some extent can be read independently of each other, grouped by the authors under six headings: (1) subideals and "coalescent" classes of Lie algebras; (2) the Mal'cev correspondence between locally nilpotent Lie algebras and certain locally nilpotent groups, along with a study of various locally nilpotent radicals; (3) finiteness conditions, especially minimal and maximal conditions on subideals; (4) properties of finitely generated solvable Lie algebras suggested by P. 